\title{
O uso de softwares e plataformas on-line no ensino da Matemática
}

\author{
The use of online software and platforms in Mathematics teaching \\ El uso de software y plataformas en línea en la enseñanza de las Matemáticas
}

Recebido: 18/08/2021 | Revisado: 22/08/2021 | Aceito: 24/08/2021 | Publicado: 25/08/2021

Marco Aurélio Calixto Ribeiro de Holanda
ORCID: https://orcid.org/0000-0002-0860-2458
Universidade Federal de Pernambuco, Brasil
E-mail: marco.calixto@ ufpe.br
Willames de Albuquerque Soares
ORCID: https://orcid.org/0000-0003-3268-7241
Escola Politécnica de Pernambuco, Brasil
E-mail: was@ poli.br

\begin{abstract}
Resumo
A matemática sempre foi uma das disciplinas de maiores queixas entre os alunos, por estes apresentarem dificuldades no aprendizado de temas básicos, seja do ensino fundamental ou superior. Uma grande problemática que tem levado os alunos enxergarem a matemática dessa maneira é o tipo de metodologia empregada no ensino. Por conta disso, o ensino da matemática tem sido amplamente discutido em diversas pesquisas, de modo que, com a pandemia causada pelo COVID, tem ampliado as discussões sobre o uso de metodologias de ensino. Deste modo, objetiva-se mostrar que o uso dos softwares e das plataformas on-line podem ser ferramentas importantes no ensino da matemática, justamente por relacionarem exemplos práticos e visuais à teoria lecionada em sala de aula. A partir da análise de outras pesquisas que utilizaram essas tecnologias no âmbito educacional, constatou-se que o ensino da matemática pode ser tratado de diferentes maneiras entretanto, as metodologias tradicionais de aprendizagem não são tão eficientes quanto os que utilizam ferramentas práticas e visuais.
\end{abstract}

Palavras-chave: Freeware; Winplot; WolfranAlpha.

\begin{abstract}
Mathematics has always been one of the subjects with the biggest complaints among students, as they have difficulties in learning basic topics, whether in elementary or higher education. A major issue that has led students to see mathematics in this way is the type of methodology used in teaching. Because of this, the teaching of mathematics has been widely discussed in several researches, so that, with a pandemic caused by COVID, it has expanded as applicable to the use of teaching methodologies. Thus, the objective is to show that the use of software and online platforms can be important tools in the teaching of mathematics, precisely because they relate practical and visual examples to the theory taught in the classroom. From the analysis of other researches that use technologies in the educational field, it was found that the teaching of mathematics can be treated differently, however, as traditional learning methodologies are not as efficient as those that use practical and visual tools.
\end{abstract}

Keywords: Freeware; Winplot; WolfranAlpha.

\section{Resumen}

La matemática siempre ha sido una de las asignaturas con mayores quejas entre los estudiantes, ya que tienen dificultades para aprender temas básicos, ya sea en la educación primaria o superior. Un tema importante que ha llevado a los estudiantes a ver las matemáticas de esta manera es el tipo de metodología utilizada en la enseñanza. Por ello, la enseñanza de las matemáticas ha sido ampliamente discutida en varias investigaciones, por lo que, con la pandemia provocada por COVID, se han ampliado las discusiones sobre el uso de metodologías de enseñanza. Así, el objetivo es mostrar que el uso de software y plataformas online pueden ser herramientas importantes en la enseñanza de las matemáticas, precisamente porque relacionan ejemplos prácticos y visuales con la teoría impartida en el aula. A partir del análisis de otras investigaciones que utilizaron estas tecnologías en el campo educativo, se encontró que la enseñanza de las matemáticas puede ser tratada de diferentes formas, sin embargo, las metodologías de aprendizaje tradicionales no son tan eficientes como las que utilizan herramientas prácticas y visuales.

Palabras clave: Freeware; Winplot; WolfranAlpha.

\section{Introdução}

As operações e expressões matemáticas estão presentes em situações corriqueiras do dia a dia, de modo que elas acabam sendo utilizadas em mercados, escolas, estudos científicos, sensos e pesquisas públicas, e em outras soluções de 
problemas cotidianos. Suas aplicações são, por muitas vezes, despercebidas pelos seus usuários por estarem foram do âmbito educacional (Castro; Pereira, 2020).

Deste modo, a desconstrução dos padrões piagetianos brasileiros, originados na década de 70 (Vasconcelos, 1997), pode ser um forte aliado dos educadores na transmissão do conhecimento, principalmente no que diz respeito a matemática, por muitos alunos terem algum tipo de dificuldade no seu entendimento. Alguns autores, como Souza e Fraiha-Martins (2019) propuseram o uso de ferramentas digitais e tradicionais da cultura oriental (ferramentas visuais), como o origami, no ensino da geometria.

Entretanto, vale salientar que o uso da tecnologia pode ser prejudicial no ensino, principalmente quando não é utilizada como uma ferramenta e sim como a fonte principal do conhecimento (Flanagan, 2008). Por isso, os cursos de educação a distância são pensados e montados por professores que estão adequando o conhecimento a uma nova abordagem, seja ela através de vídeos, textos ou jogos (Barbosa; Pontes; Castro, 2020; Santo; Santos, 2020). Silva, Santos e Soares (2012) ainda ressaltam que o ensino de Matemática passa por diversas fases de desenvolvimento, sofreu e permanece sofrendo transformação para se adaptar ao contexto social de cada época. Atualmente as tecnologias computacionais se fazem cada vez mais presentes no cotidiano da população. No contexto atual o computador, inicialmente utilizado para entreter, facilitar e melhorar as vidas das pessoas quando estendido ao campo da Educação Matemática pode ser uma importante ferramenta didática, possibilitando aos alunos e professores o desenvolvimento de uma aprendizagem mais significativa. (Silva; Santos; Soares; 2012, p. 1).

Para Holanda, Manfredi e Silva (2020, p. 2) no âmbito escolar, "é essencial que tais dispositivos estejam disponíveis no ambiente estudantil e que os aparelhos de uso próprio do aluno, também sejam considerados nas metodologias pedagógicas". Por isso, o uso de softwares e plataformas on-line como o Excel, o PowerPoint, Winplot, WolframAlpha, Canvas, Powtoon, entre outros, principalmente aqueles que podem ser instalados ou utilizados em aparelhos celulares ou tabletes, vem sendo amplamente utilizados por educadores como ferramentas de auxílio.

A partir das diretrizes listadas na Base Nacional Comum Curricular (BNCC) é possível inferir que os professores de matemática podem implementar o uso dessas tecnologias como parte de sua metodologia de ensino, tornando os conteúdos desta disciplina mais acessível por tratarem com uma conotação cotidiana ou visual que façam mais sentido (Afonso; Maldonado, 2020). Além disso, Os Parâmetros Curriculares Nacionais para o Ensino Médio já preveem o influxo das Tecnologias de Informação e Comunicação no ensino de disciplinas como a Matemática (Amancio; Sanzovo, 2020).

Além dos softwares, outras tecnologias tem sido empregadas, como o uso do eye-tracking na análise da influência do uso das cores como uma ferramenta visual no ensino das Ciências e Matemática (Oliveira; Locatelli; Sato, 2021). Esses autores analisaram estudos publicados entre os anos de 2005 e 2020, os quais mostraram que esse tipo de abordagem, do uso das cores, tem sido amplamente utilizado no ensino superior, mas apenas um único trabalho trazia uma aplicação para o ensino médio, mostrando a escassez no uso de ferramentas. Além disso, os futuros profissionais da área das ciências biológicas são os que mais fazem uso destas ferramentas visuais, por meio do elo entre as imagens e o conteúdo textual formal.

A tecnologia, através das ferramentas visuais, tem proporcionado uma redução do distanciamento entre o aluno e o conhecimento, uma vez que os educadores podem utiliza-la para ajudar na transmissão do conhecimento, melhorando a qualidade do ensino. Além disso, elas podem alavancar a curva de aprendizado dos alunos, fazendo com que eles aprendam cada vez mais rápido, pois esses artifícios visuais, trazidos pela tecnologia, auxiliam, principalmente, na fixação do conteúdo (Ribeiro; Paz, 2012).

Visando avaliar os benefícios trazidos pela tecnologia no ensino da Matemática, seja na interpretação dos caracteres escritos, dos cálculos realizados e na fixação dos conceitos, Perius (2012) utilizou vídeos e softwares no ensino de alunos do Ensino Fundamental. Com isso, o autor constatou que a aplicação dessas ferramentas facilitou aos alunos a compreensão e o 
domínio da linguagem matemática.

Deste modo, por relacionarem exemplos práticos e visuais à teoria lecionada em sala de aula, objetiva-se mostrar que o uso dos softwares e das plataformas on-line podem ser ferramentas importantes no ensino da matemática, seja no ensino fundamental ou superior.

\section{Metodologia}

A metodologia deste trabalho consiste no uso de softwares e plataformas on-line, os quais tem se tornados aliados dos professores no que diz respeito ao repasse do conhecimento (Flanagan, 2008), haja vista que eles são ferramentas que podem auxiliar o professor a tornar o conteúdo mais acessível aos alunos, principalmente para os que demonstram alguma dificuldade de aprendizado. Deste modo, nesta sessão serão apresentados alguns softwares e plataformas gratuitas (freewares), ou de fácil acesso, que podem ser utilizadas pelos professores de matemática ou alunos, de diversas áreas e níveis (ensino fundamental, ensino superior, educação de jovens e adultos e etc.), que precisem realizar trabalhos de diversas naturezas.

\subsection{Excel}

O Excel é um software criado pela Microsoft Corporation para organização e análise numérica em planilhas, o qual possibilita ao usuário realizar diversas operações básicas, como soma, subtração, multiplicação e divisão, e operações mais complexas como o cálculo de medidas de dispersão (média, moda e mediana) e até mesmo a resolução de expressões algébricas, seja do primeiro e segundo grau ou polinomial. Abreu et al. (2002) ainda ressaltam que

$\mathrm{O}$ uso de planilhas eletrônicas no ensino da álgebra é particularmente interessante porque permite que o aluno se envolva num processo interativo de resolução ou modelação de um determinado problema. A sua utilização pode ser associada com essas abordagens metodológicas, a resolução de problemas ou a Modelagem Matemática. (Abreu et al., 2002, p.92).

A ferramenta também possibilita ao usuário a confecção e análise de gráficos de diferentes tipos (de dispersão com e sem linhas, de bolhas, de pizza bi ou tridimensional, de barras, de caixas, entre outros). Software é disponibilizado para computadores, que utilizam o Windows e o macOS como sistemas operacionais, e para dispositivos móveis e o seu layout pode ser observado na Figura 1. 
Figura 1 - Layout de abertura do Excel.

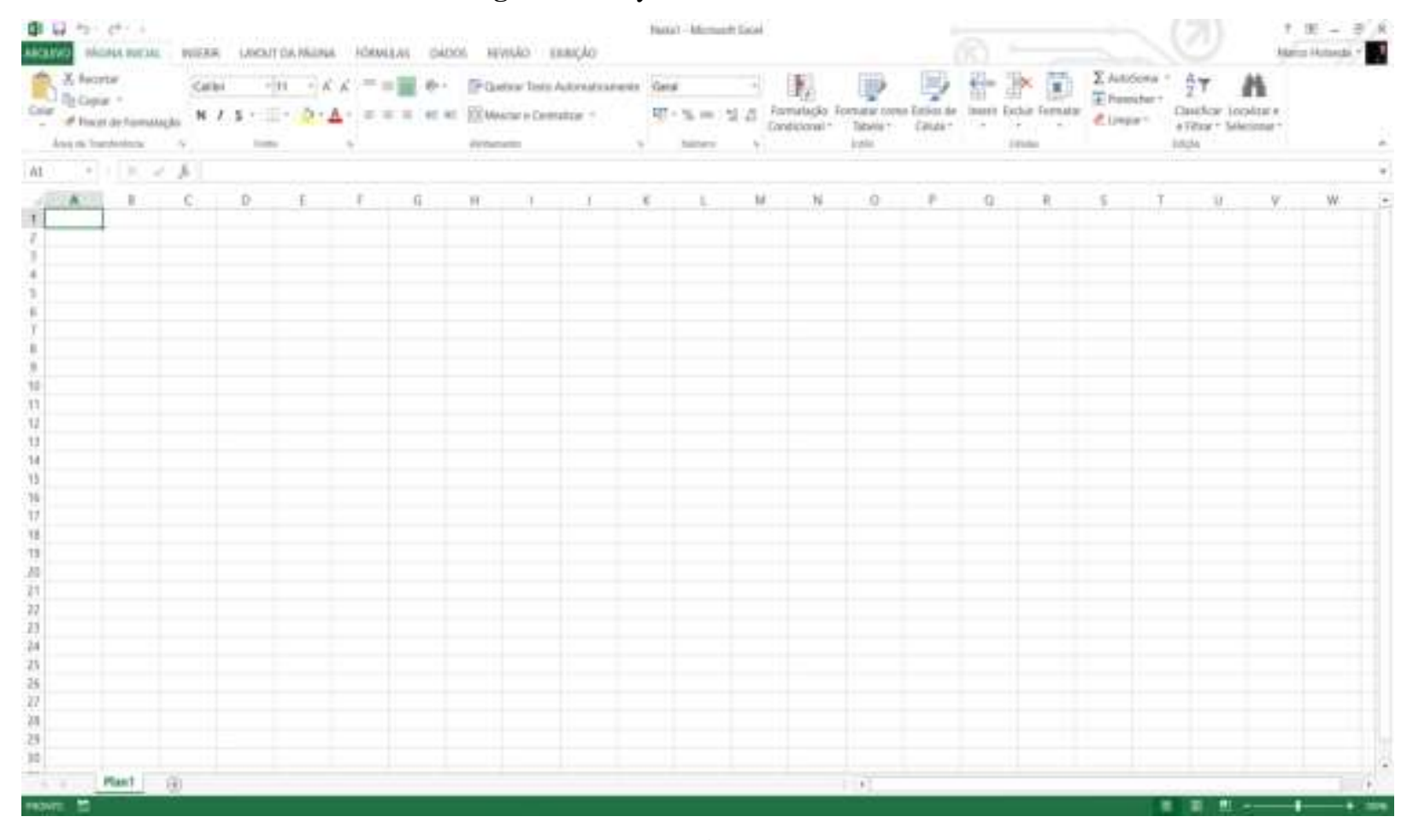

Fonte: Autores.

\subsection{WolframAlpha}

O WolframAlfa é uma plataforma on-line idealizada pelo visionário cientista Stephen Wolfram (Wolfram, 2016), a qual é utilizada no cálculo de derivadas e integrais, de finanças, para o clima, encontrar objetos no céu, conversão de tempo e datas, entre outros. O WolframAlpha foi montado a partir de outro produto desenvolvido por Stephen, o Matehmatica (WolframAlpha, 2021), o qual é amplamente utilizado por estudantes e professores para análise de dados, estudos geométricos e de imagens, estudo de grafos, manipulação de expressões algébricas e etc.

Além de ser uma plataforma interativa, um dos principais diferenciais do WolframAlpha é que não são disponibilizados apenas os resultados, mas também o modo como o cálculo foi realizado, através do recurso "Step-by-Step" (Rosly; Abdullah; Shukri, 2020), o qual também é disponibilizado por plataformas afins, como o Symbolab. Por exemplo: na solução de uma integral indefinida, ele também fornece ao usuário o passo a passo de como o cálculo foi realizado, tornando-o uma poderosa ferramenta de estudos para alunos de exatas. O layout do WolframAlpha pode ser observado na Figura 2. 
Figura 2 - Visão da página de abertura do WolframAlpha.

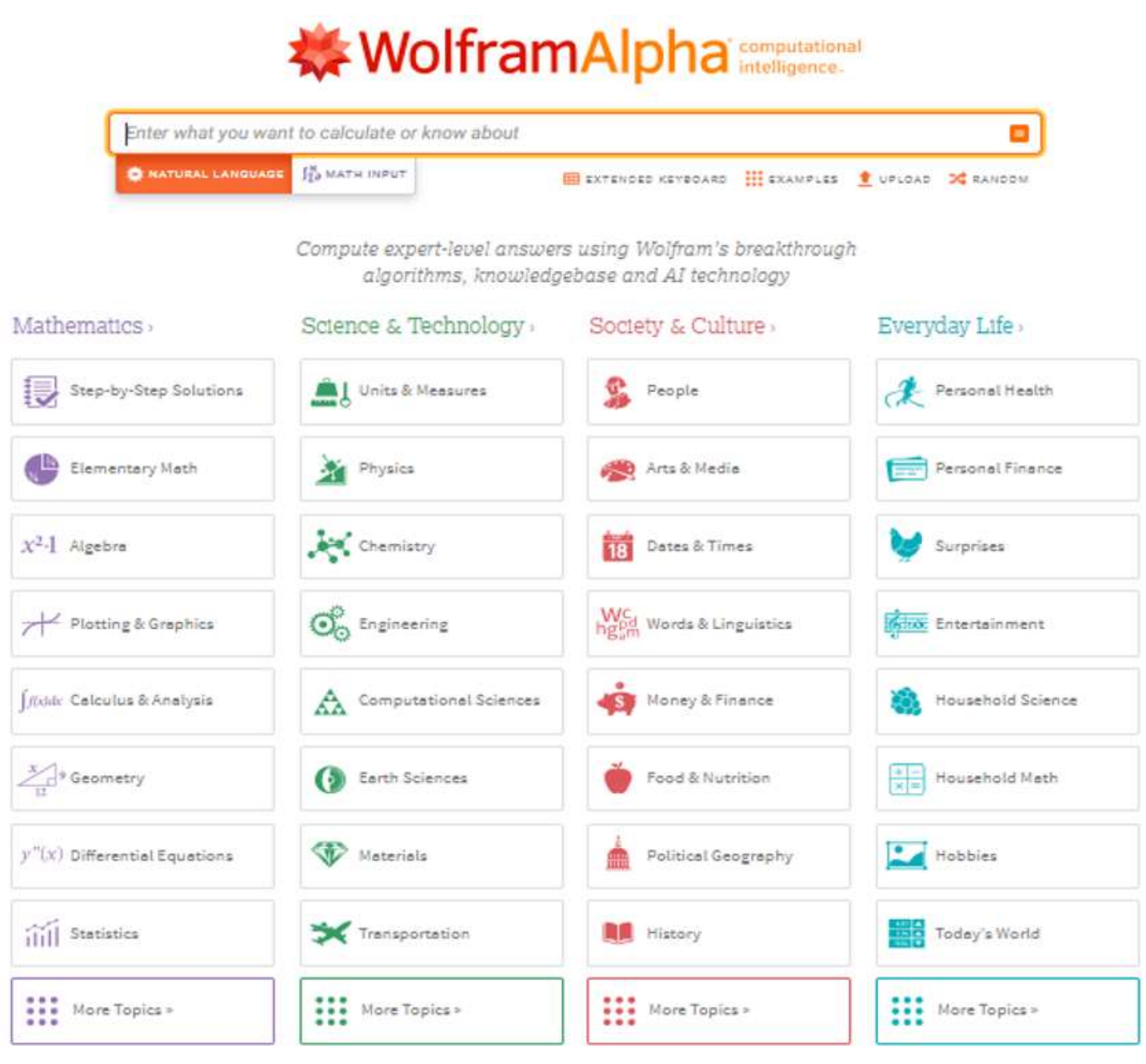

Fonte: Autores.

\subsection{Winplot}

O Winplot é um software gratuito, feito em C++ pelo professor Richards Parris, da Philips Exeter Academy, utilizado para a confecção de gráficos bi ou tridimensionais (Novais, 2021) e pode ser utilizado como facilitador do processo de aprendizagem (Romeiro; Garcia; Romão, 2021). O Winplot é de fácil utilização pois há uma aparência aprazível, além de fornecer ajuda em todas as suas partições, o que o faz ser considerado intuitivo pela maioria dos usuários. Além disso, esse programa possibilita ao usuário selecionar qual a língua que será utilizada na interface, de modo que o Português figura entre as opções. Esse programa foi desenvolvido para Windows, mas também funciona em outros sistemas operacionais gratuitos, como o Linux. O layout do software pode ser observado na Figura 3 abaixo. 
Figura 3 - Tela inicial do Winplot para gráficos bidimensionais.

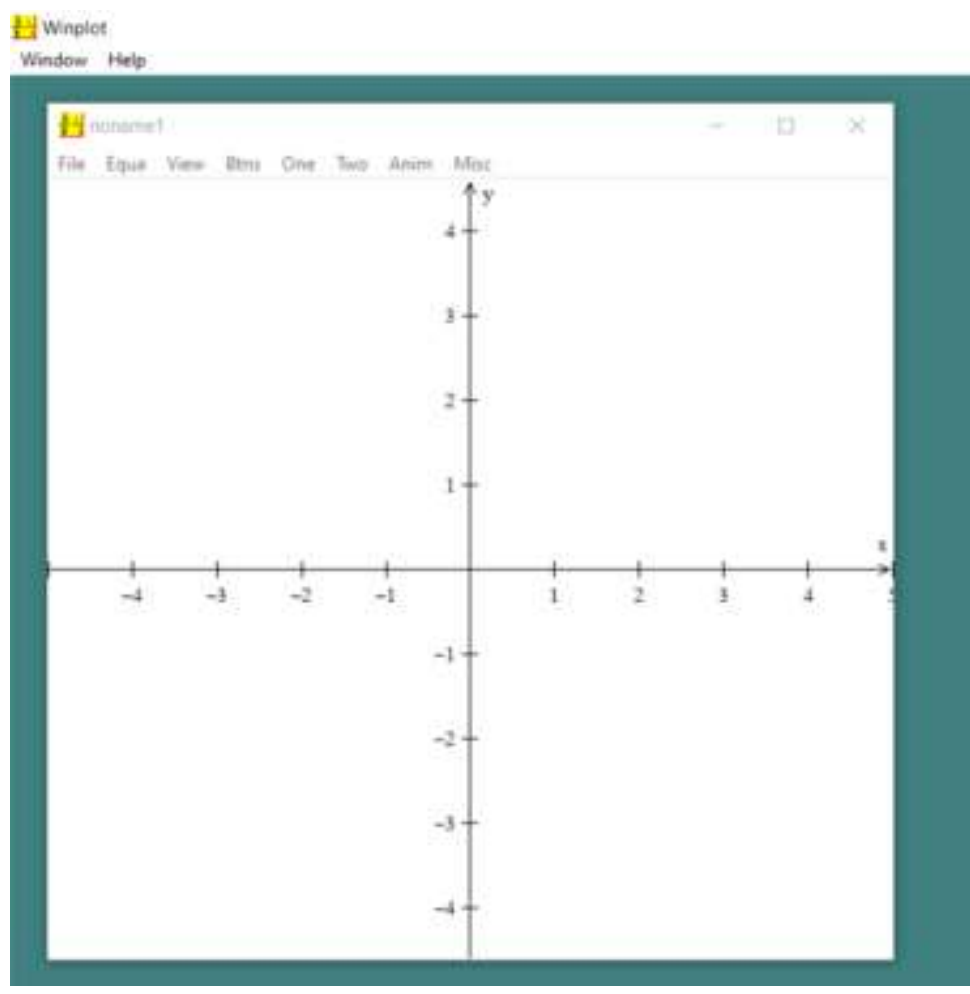

Fonte: Autores.

\section{Resultados e Discussão}

Diversos métodos tem sido utilizados pelos professores de matemática, em sala de aula, para facilitar a compreensão do aluno. Novais (2021) propôs o uso do winplot na aplicação de funções de primeiro grau. Tal estudo pode ser utilizado como referência por professores do ensino fundamental como uma aplicação visual das equações de primeiro grau, quando este conteúdo estiver sendo ministrado. Esse estudo de Novais (2021) ainda traz uma série de passos que podem ser seguidos pelo professor, caso este ainda não esteja familiarizado com o software, bem como um quadro mostrando o modo como as expressões devem ser escritas, Figura 4.

Figura 4 - Tabela de funções e suas respectivas sintaxes usadas no Winplot.

\begin{tabular}{|c|c|c|c|c|c|c|c|c|c|}
\hline Função & \multicolumn{1}{|c|}{$a+b$} & $a-b$ & \multicolumn{2}{|c|}{$a \cdot b$} & \multicolumn{2}{c|}{$\frac{a}{b}$} \\
\hline Sintaxe & \multicolumn{1}{|c|}{$a+b$} & \multicolumn{2}{|c|}{$a-b$} & \multicolumn{2}{|c|}{$a^{*} b$} & \multicolumn{2}{c|}{$a / b$} & \\
\hline Função & $x^{n}$ & $a^{x}$ & $\log x$ & $\ln x$ & $\sqrt[n]{x}$ & $|\mathrm{x}|$ & $\operatorname{sen} x$ & $\cos x$ & $\tan x$ \\
\hline Sintaxe & $x^{\wedge} n$ & $a^{\wedge} x$ & $\log (x)$ & $\ln (x)$ & $\operatorname{root}(n, x)$ & $a b s(x)$ & $\sin (x)$ & $\cos (x)$ & $\tan (x)$ \\
\hline
\end{tabular}

Fonte: Novais (2021)

Silva, Santos e Soares (2012) utilizaram o Winplot como uma ferramenta de auxílio ao ensino e a aprendizagem da Matemática. Neste caso, os autores avaliaram o uso tanto com alunos no ensino médio quanto com alunos do ensino superior da Universidade de Pernambuco, os quais cursavam Licenciatura em Matemática. Para isso, eles propuseram algumas atividades, incluindo a resposta a questionários e o uso do software.

Neste caso, Silva, Santos e Soares (2012) notaram que o Winplot contribuiu significativamente para o 
desenvolvimento das atividades propostas, pois o dinamismo que o ele confere dificilmente poderia ser reproduzido se a atividade fosse realizada do modo tradicional. Além disso, foi notório um empenho dos alunos participantes, pois tratava-se de uma abordagem diferente para a trivial construção de gráficos bidimensionais (Silva, Santos e Soares, 2012).

Romeiro, Garcia e Romão (2021) ainda trazem uma outra aplicação para o uso do Winplot. Neste caso, os autores sugerem o uso desta ferramenta matemática no ensino da física, uma vez que diversas situações o professor de física necessita desenhar o gráfico que representa uma trajetória, seja de um projétil, de um carro ou de uma pessoa, ou seja, para representar Movimentos Uniformes (MU), seja durante a explicação do conteúdo ou na resolução de exercícios.

Além disso, Romeiro, Garcia e Romão (2021) mostram que os resultados das questões resolvidas podem ser expressos em gráficos de barras, os quais podem ser facilmente confeccionados utilizando-se o auxílio do Excel. Com isso, esses autores mostram que as ferramentas supracitadas facilitam o processo de aprendizagem dos alunos, principalmente quando o conhecimento ou o conteúdo está sendo repassado pela primeira vez, corroborando assim com o que foi observado por Silva, Santos e Soares (2012) ao analisarem a facilidade com a qual o conhecimento foi repassado e absorvido.

Silva, Santos e Soares (2012) ainda afirmam que a correlação entre conteúdos teóricos e o demonstrativo prático estimulam o desenvolvimento do raciocínio, de modo que o Winplot pode ser uma dessas ferramentas práticas, principalmente durante o estudo da trigonometria. Atualmente, muitas instituições que oferecem ensinos a distância (EAD) já trabalham com a ideia da gamificação, onde há softwares com os quais os alunos podem interagir e praticar o que foi aprendido em aula (Pimentel; Ferreira; Freitas, 2020; Ribeiro et al., 2020).

Já Lopes e Bisognin (2015) utilizaram o Excel como ferramenta de ensino de funções matemáticas para alunos do Ensino Fundamental. Esses autores constataram que o uso de recursos pedagógicos não convencionais, como o uso de softwares, pode ser desafiador, principalmente para o educador. Entretanto, foi possível notar que o uso do Excel propiciou aos alunos um estímulo à comunicação, desenvolveu diferentes formas de raciocínio, estimulou diferentes habilidades e os levou a reflexão sobre os temas propostos em sala (Lopes; Bisognin, 2015).

Visando analisar as contribuições feitas pelo uso das Tecnologias da Informação e Comunicação na aprendizagem da Estatística, e promover o seu ensino como uma forma impulsionadora para o desenvolvimento crítico dos alunos, Camillo (2017) apresentaram o Excel à alunos do $5^{\circ}$ ano do Ensino Fundamental. Como a Estatística não é um conteúdo comumente lecionado para alunos dessa faixa etária, a autora enfatiza que o professor deve especializar-se para repassar este conhecimento de maneira que o aluno não apresente grandes dificuldades no seu aprendizado e se afaste da disciplina ou crie algum tipo de bloqueio.

Ao se depararem com quatro perguntas propostas por Camillo (2017), sobre ter ou não computador, ter conhecimento da existência ou não do Excel, ter utilizado esse software em alguma outra aula e se eles gostariam de conhecer e utilizar outros softwares em sala, os alunos responderam conforme mostrado na Figura 5. Onde é possível observar que mesmo quase $33 \%$ da turma já tivesse ouvido falar do Excel, 100\% deles nunca o utilizou em sala, e $98 \%$ deles gostariam de ter acesso a outros softwares como este. 
Figura 5 - Respostas dos alunos às quatro perguntas realizadas.

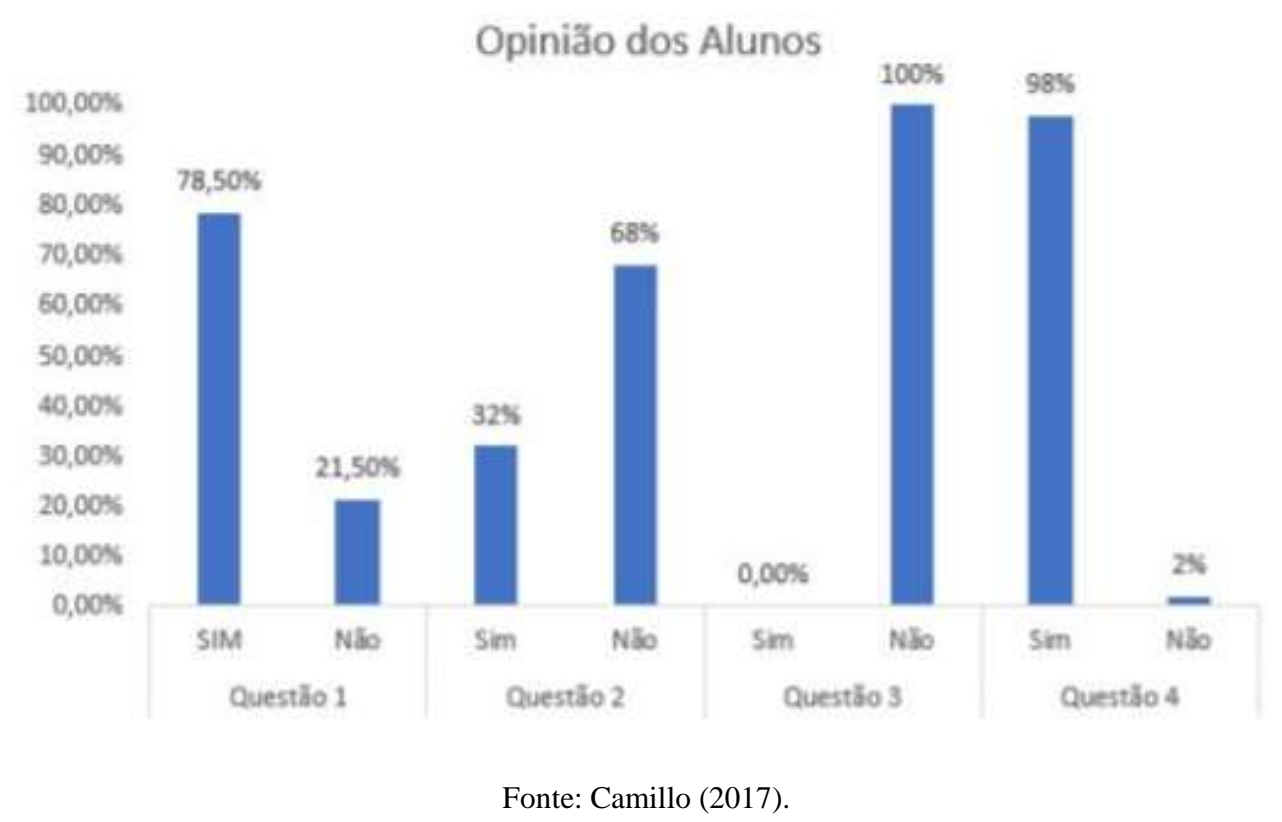

Ademais, o Excel também pode ser utilizado por pesquisadores da área pedagógica, bem como foi feito por Castro e Pereira (2020) ao estudarem como é realizado o ensino da matemática em escola públicas de Minas Gerais e se o cotidiano é levado em consideração. Neste caso, os autores utilizaram o Excel para analisar os dados que foram coletados a partir de uma pesquisa virtual, onde os participantes respondiam um questionário que era feito.

Além de conteúdos simples, como funções de primeiro e segundo grau, as plataformas e softwares disponíveis facilitam também os ensinamentos do cálculo, onde os alunos podem conferir suas contas utilizando calculadoras de limites, derivadas e integrais, bem como é disponibilizado no Wolframalpha e no Symbolab por exemplo, (Rosly; Abdullah; Shukri, 2020). Plataformas como estas ainda permitem que o aluno observe o passo a passo da resolução de um problema, diferentemente de calculadoras científicas gráficas comuns que lhes conferem apenas o resultado final e possuem um alto valor de aquisição, o que favorece a compreensão e o aprendizado (Figura 6). 
Figura 6 - Resolução da integral indefinida do sen2(x) com o passo a passo, no Symbolab.

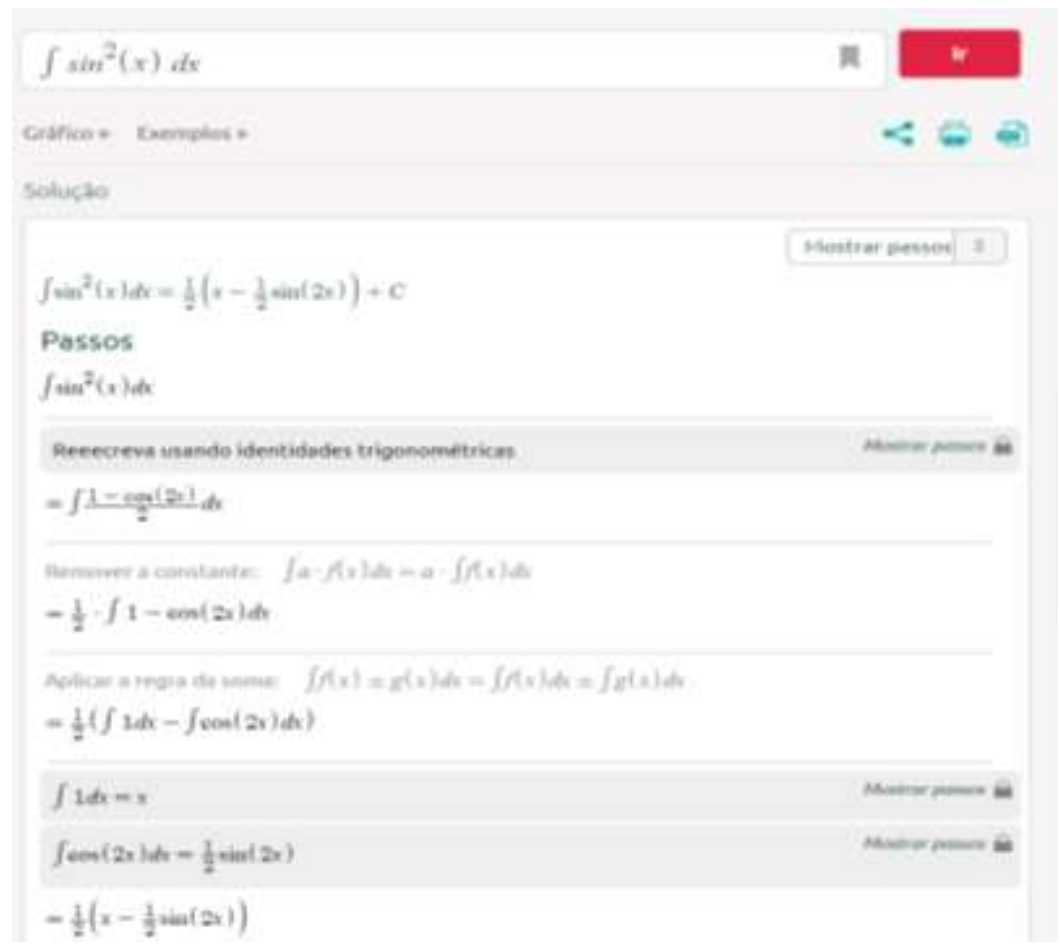

Fonte: Autores.

Outros autores como Resende e Martins (2018) mostraram que o Wolframalpha é uma ferramenta que possui um elevado potencial pedagógico no ensino da matemática, uma vez que pode ser utilizado na análise de gráficos, na geometria analítica, no cálculo diferencial e integral e etc, corroborando com o que foi evidenciado por Lopes e Bisognin (2015) e por Silva, Santos e Soares, 2012 ao aplicarem o Excel no ensino da matemática.

Além disso, Resende e Martins (2018) ainda mostraram que esta plataforma possui diversas vantagens com relação aos métodos tradicionais de ensino e aprendizagem, as quais são imprescindíveis para o ensino a distância, pois os alunos que procuram por esta modalidade possuem características distintas daqueles que frequentam o ensino usual e, por isso, pensam de modo diferente e tem outras exigências.

Gayoso et al. (2020) ainda ressaltam que após o tratado de Bologna, o qual trata da unificação do ensino universitário europeu e que foi assinado por 49 países, o uso de ferramentas computacionais como softwares e plataformas on-line, que propiciem ao professor mostrar exemplos mais semelhantes a realidade, é de fundamental importância. De modo que, estes exercícios podem ser peças cruciais na construção do conhecimento dos alunos.

\section{Conclusão}

O ensino da matemática pode ser tratado de diferentes maneiras entretanto, os modelos tradicionais de aprendizagem, onde o aluno só observa o que é feito e apenas aceita o que lhe foi repassado, sem visualizar ou tornar palpável aquilo que foi aprendido, acabam gerando dúvidas.

Além disso, muitos alunos relatam não terem afinidade pela matemática justamente por nunca terem tido contato com ferramentas visuais como o Winplot, o Excel, o Wolframalpha, entre outras. Se o professor busca alternativas para facilitar a compreensão dos alunos a um determinado conteúdo, ele estará desmistificando a matemática e tornando seu ensinamento mais aprazível para muitos deles, principalmente para os que julgavam não ter aptidão para essa disciplina.

Como sugestões, é possível sugerir que estas plataformas e softwares sejam colocados em prática nas salas de aula, e 
um questionário pode ser aplicado. Neste caso, os alunos responderiam como o uso destas metodologias de visualização ajudou no seu aprendizado e se desta maneira foi mais aprazível.

\section{Referências}

Abreu, M. A. M. (2002). Metodologia de Ensino de Matemática. LED.

Afonso, T. \& Maldonado, M. (2020). Um exemplo prático do uso da tecnologia como meio para o ensino de matemática. Proceeding Series of the Brazilian Society of Computational and Applied Mathematics, 7(1), p. 1-2.

Amancio, D. T. \& Sanzovo, D. T. (2020). Ensino de Matemática por meio das tecnologias digitais. Revista Educação Pública, $20(47)$, p. 1-5.

Barbosa, F. E., Pontes, M. M. \& Castro, J. B. (2020). A utilização da gamificação aliada às tecnologias digitais no ensino da matemática: um panorama de pesquisas brasileiras. Revista Prática Docente, 5(3), 1593-1611.

Camillo, C. M. (2017) O Uso do Software Excel no ensino da Matemática. Acesso Livre, 7, 199-206.

Castro, B. P. \& Pereira, A. A. S. (2020). Educação matemática no ensino fundamental: matemática de caráter prático nos anos iniciais. Revista Científica UNIFAGOC, 1, p. 140-150.

Flanagan, J. L. (2008). Technology: The Positive And Negative Effects On Student Achievement (Dissertação de mestrado). Mestrado in Education and Human Development - State University of New York College.

Holanda, T., Manfredi, V. \& Silva, R. (2020). Tecnologia e ensino da matemática dos anos iniciais do ensino fundamental: o uso de computadores, softwares e vídeos na otimização da prática educativa. Anais do Congresso Internacional de Educação e Tecnologias - Encontro de Pesquisadores em Educação a Distância, São Carlos, SP, Brasil. https://cietenped.ufscar.br/submissao/index.php/2020/article/view/1419.

Lopes, E. J. S. \& Bisognin, C. (2015). O uso do Excel como ferramenta no ensino de funções afins. (Monografia de Especialização em Matemática, Mídias Digitais e Didática). Universidade Federal do Rio Grande do Sul, Instituto de Matemática. Rio Grande do Sul, Brasil.

Novais, M. J. C. (2021). Winplot: aplicações de funções de $1^{\circ}$ grau. (Monografia de Licenciatura em Matemática). Pontifícia Universidade Católica de Goiás. Goiânia, Goiás, Brasil.

Oliveira, V. M., Locatelli, S. W. \& Sato, J. R. (2021). Influência das cores no contexto educacional de Ciências e Matemática: uma revisão de literatura sobre a utilização de eye-tracking. Amazônia - Revista de Educação em Ciências e Matemática,17(38), p. 244-266.

Perius, A. A. B. (2012). A tecnologia aliada ao ensino da Matemática. (Monografia de Especialização em Mídias na Educação). Universidade Federal do Rio Grande do Sul, Centro Interdisciplinar de Novas Tecnologias na Educação. Cerro Largo, Rio Grande do Sul, Brasil.

Pimentel, F. S. C., Ferreira, A. R. \& Freitas, R. O. (2020) Gamificação como estratégia pedagógica no combate à evasão: Potencialidades da implementação no ensino superior. Anais do Congresso Internacional de Educação e Tecnologias - Encontro de Pesquisadores em Educação a Distância, São Carlos, SP, Brasil. https://cietenped.ufscar.br/submissao/index.php/2020/article/view/1266.

Resende, M. A. \& Martins, L. G. (2018). Wolfram|Alpha: um mecanismo de busca no auxílio do processo de ensino-aprendizagem de conteúdos de Matemática na educação a distância. BoEM, 6(11), p. 432-448.

Ribeiro, F. M. \& Paz, M. G. (2012). O ensino da matemática por meio de novas tecnologias. Revista Modelos - FACOS / CNEC Osório, 2 (2), p. 12-21.

Ribeiro, V. G., Zabadal, J., Trommer, T., Silveira, A. L. M., Silveira, S. R., Bertoloni, C., Cunha, G. B. \& Bigolin, N. M. (2020). Emprego de Técnicas de Gamificação na Educação Científica: relato de uma intervenção como apoio à Estatística. Research, Society and Development, 9(1), 1-26.

Romeiro, R. A. G., Garcia, R. V. \& Romão, E. C. (2021). O ensino de funções e a educação tecnológica: o simulador PHET e o software Winplot como facilitadores da aprendizagem. Caminhos da educação matemática em revista, 11(2), p. 111-131.

Rosly, W. N. S. W. M., Abdullah, S. S. S. \& Shukri, F. N. A. (2020). The uses of Wolfram Alpha in Mathematics. Articles of Teaching and Learning in Higher Education, 1, p. 96-103.

Santo, E. E. \& Santos, W. S. (2020). A gamificação como elemento potencializador de um modelo pedagógico para a educação a distância. Anais do Congresso Internacional de Educação e Tecnologias - Encontro de Pesquisadores em Educação a Distância, São Carlos, SP, Brasil. https://cietenped.ufscar.br/submissao/index.php/2020/article/view/1167.

Silva, A. C., Santo, L. V. \& Soares, W. A. (2012). Utilização do Winplot Como Software Educativo Para o Ensino de Matemática. Revista Diálogos, 6, p. 187206.

Souza, J. K. C. \& Fraiha-Martins, F. (2019). Origami e tecnologias digitais: instrumentos mediadores para o ensino de geometria nos anos iniciais do ensino fundamental. Revista Tecnologia Educacional, 226, p. $42-54$.

Vasconcelos, S. J. S. (1997). Raízes e caminhos do pensamento piagetiano no Brasil. In: Freitag, B. (Org.) Piaget: 100 anos (pp. 193-210). Cortez.

Wolfram, S. (2016). Idea Makers: Personal Perspectives on the Lives \& Ideas of Some Notable People: Wolfram Media, Inc.

Wolframalpha Llc, (2021, julho 27) WolframAlpha computational intelligence. http://www.wolframalpha.com. 